\title{
Glycine supplementation reduces the severity of chemotherapy-induced oral mucositis in hamsters
}

\author{
Odara Maria de Sousa Sá ${ }^{1}$, Nilza Nelly Fontana Lopes ${ }^{2}$, Maria Teresa Seixas Alves ${ }^{3}$, \\ Rajesh V. Lalla ${ }^{4}$, Maria Luiza Vilela Oliva ${ }^{5}$, Eliana Maria Monteiro Caran ${ }^{6}$ \\ ${ }^{1}$ Department of Pediatrics, Federal University of São Paulo, São Paulo, Brazil; odarasousa@yahoo.com.br \\ ${ }^{2}$ Former Head Division of Dentistry, Pediatric Oncology Institute, São Paulo, Brazil; nnflopes@terra.com.br \\ ${ }^{3}$ Department of Pathology, Federal University of São Paulo, São Paulo, Brazil; mtseixas@patologia.epm.br \\ ${ }^{4}$ Section of Oral Medicine, University of Connecticut Health Center, Farmington, USA; Lalla@uchc.edu \\ ${ }^{5}$ Department of Biochemistry, Federal University of São Paulo, São Paulo, Brazil; olivaml.bioq@epm.br \\ ${ }^{6}$ Department of Pediatrics, IOP/GRAACC Medical School of Federal University of São Paulo, São Paulo, Brazil; \\ elianacaran@terra.com.br
}

Received 29 June 2013; revised 29 July 2013; accepted 7 August 2013

Copyright (C) 2013 Odara Maria de Sousa Sá et al. This is an open access article distributed under the Creative Commons Attribution License, which permits unrestricted use, distribution, and reproduction in any medium, provided the original work is properly cited.

\begin{abstract}
Objective: Oral mucositis (OM) is a devastating toxicity associated with cytotoxic cancer therapy. The OM pathogenesis and the complex interactions occur in response to tissue insult. Application of this evolving model has aided in the development of mechanistically based therapies for the prevention and treatment of mucositis. The present study was to assess the effects of glycine supplementation on chemotherapy-induced oral mucositis. Methods: In a hamster cheek pouch model of chemotherapyinduced oral mucositis, one group of 20 animals received systemic glycine supplementation for 7 days, while another similar control group did not. Clinical mucositis severity and neutrophil infiltrate (on histology) were assessed by blinded examiners. Free radical production was measured as malondialdehyde (MDA) levels. Results: As compared to control animals, glycine-treated animals demonstrated a highly significant reduction in clinical severity of oral mucositis, neutrophil infiltrate, and MDA levels $(p<0.001$ for all). Conclusions: Glycine supplementation reduces the severity of chemotherapy-induced oral mucositis in an animal model. This effect is at least partly mediated through inhibition of the inflammatory response and reduced production of damaging free radicals.
\end{abstract}

Keywords: Glycine; Chemotherapy; Oral Mucositis; Neutrophils; Malondialdehyde; Inflammatory

\section{INTRODUCTION}

Oral mucositis (OM) presents as erythematous and ulcerative lesions of the oral mucosa, secondary to chemotherapy and/or radiation therapy for cancer [1-3]. Clinical manifestations of OM include intense pain, interfereence with ingestion of food and drink, and impaired communication. Moreover, infection associated with oral mucosal lesions can progress to life threatening sepsis during periods of intense immunosuppression [4]. OM impacts negatively on patients' survival and quality of life, and is associated with longer hospitalizations and higher costs $[5,6]$. Perhaps most importantly, dose-reductions in cancer therapy due to mucositis can adversely affect the outcomes of cancer treatment. However, treatment options for OM are very limited and current management strategies are largely palliative [7].

$\mathrm{OM}$ is an inflammatory response of the oral mucosa that pathophysiology is complex and multifactorial [8]. Histopathological evaluation of mucositis lesions shows mucosal thinning, caused by apoptosis and depletion of the epithelial basal layer, with subsequent denudation and secondary bacterial infection [9]. Development of oral mucositis involves oxidative stress and the accumulation of reactive oxygen species (ROS) [10]. This oxidative stress can produce lipid peroxidation and inflammation [11]. The inflammatory response in oral mucositis in- 
volves the activation of $\mathrm{NF}-\kappa \mathrm{B}$ and the upregulation of inflammatory cytokines, including TNF- $\alpha$ [12] Glycine, a simple amino acid, has been shown to have anti-inflammatory, immunomodulatory and cytoprotective effects [13]. Experimental studies have shown its protective effect on inflammatory lesions in various models. For example, glycine is a strong inhibitor of resident liver macrophages and acts via a glycine-gated chloride channel, which subsequently inhibits Kupffer cell (KC) activation by decreasing calcium inflow [14,15]. Moreover, glycine is an essential component of glutathione, which is needed for detoxification processes. In addition, glycine has indirect effects as a free radical scavenger [16].

Glycine inhibits the production of inflammatory mediators, probably by decreasing the activation of NF- $\kappa \mathrm{B}$ and TNF- $\alpha$, reducing the formation of additional free radicals and other toxic mediators, and attenuating further lipid peroxidation and glutathione depletion $[17,18]$. Recent research by Stoffels et al [19] has demonstrated the ability of glycine to reduce chemotherapyassociated injury. For example, in a clinically relevant in vivo model of chemotherapyassociated liver injury, glycine decreased liver damage (as measured by transaminases after chemotherapy), reduced microvesicular steatosis, and increased hepatic microcirculation [19].

Further, in a rat model of ischemia-reperfusion injury, glycine administration resulted in downregulation of cell apoptosis and the expression of pro-apoptotic genes [20]. Thus, glycine has been demonstrated to have positive effects on many of the pathways involved in the pathogenesis of OM. Therefore, this study was designed to assess the effects of glycine on chemotherapy-induced $\mathrm{OM}$ in a hamster model. We examined the effects of systemic glycine supplementation on the clinical severity of OM, degree of inflammatory response (by assessing neutronphil infiltrate histologically), and oxidative stress (by measuring the final product of lipid peroxidation).

\section{MATERIALS AND METHODS}

This study was approved by the Ethics Committee of São Paulo Federal University (UNIFESP) (1916/08). Forty female Golden Syrian hamsters (Mesocricetus auratus), 8 weeks old and weighing approximately $150 \mathrm{~g}$ each, were used. The animals were kept in groups of six per plastic container, with food and water available ad libitum.

\subsection{OM Induction Protocol}

A well-accepted published protocol for chemotherapyinduced oral mucositis in hamsters was used [21]. Briefly, all the animals received $80 \mathrm{mg} / \mathrm{kg}$ intraperitoneally of the chemotherapy drug 5-Fluorouracil (5-FU) on day 0 , followed by $40 \mathrm{mg} / \mathrm{kg} 5$-FUadministered intraperitoneally on day 2. The right cheek pouch of the animals was everted and the mucosa was irritated by superficial scratching with the tip of an 18-gauge needle by the same operator on days 3 and 4 .

\subsection{Glycine Supplementation}

The animals were randomly divided into two groups of 20 animals each. Animals in Group 1 received a 2 $\mathrm{mg} / \mathrm{g}$ of body weight intraperitoneal injection of Glycine (Ajinomoto, Raleigh, NC), diluted in saline at a concenration of $5 \%$. Treatment with the Glycine, was initiated on day 0 , with application once per day (in the morning), for seven days. Animals in Group 2 served as controls and did not receive any glycine supplementation but were treated identically in all other respects.

\subsection{Clinical Evaluation of OM}

Clinical evaluation of OM was performed by two blinded evaluators. On day 3 and day 7, the right cheek pouch of all animals was turned outward for the clinical evaluation of the severity of the mucositis. Mucositis scores from 0 to 5 was assigned based on the method described by Sonis et al with higher scores indicating greater severity [22] (Table 1).

\subsection{Histological Evaluation of $O M$}

All animals were sacrificed on day 7 and the right cheek pouch removed. The cheek pouch samples were labeled, immediately cooled in isopentane for $10 \mathrm{~s}$, and then flash frozen in liquid nitrogen. The fragments were positioned in such a way so as to provide cross-sectional slices during microtomy. Serial slices $(10 \mu \mathrm{m})$ were obtained in a cryostat at a temperature of $-20^{\circ} \mathrm{C}$, placed on silanized glass slides, submerged in acetone, and dried at room temperature for $10 \mathrm{~min}$. The serial sections of each sample were stained using hematoxylin-eosin staining and examined under a light microscope by a blinded pathologist. The absence or presence of microscopically visible ulceration and severity of neutrophil infiltrate were each separately scored, using the scale described by Lopes et al. [23] (Table 1).

\subsection{Measurement of Oxidative Stress: Determination of Malondialdehyde (MDA) Levels}

MDA is a final product of lipid peroxidation and a well-established measure of the level of free radicals in intestinal tissue [20,24]. To determine MDA levels, the 
Table 1. Scales used for clinical and histological evaluation of oral mucositis.

\begin{tabular}{ccl}
\hline & Grade & \multicolumn{1}{c}{ Criteria } \\
\hline & 0 & Pouch completely healthy. No erosion or vasodilatation. \\
Clinical & 1 & Erythema, but no evidence of mucosal erosion. \\
Assessment & 2 & Severe erythema, vasodilation and superficial erosion \\
& 3 & Formation of ulcers in one or more places, but not affecting more than 25\% of the surface area of the pouch. \\
& 4 & Severe erythema and vasodilation Cumulative ulcer formation about 50\% of pouch surface area. \\
& 5 & Virtually complete ulceration of the pouch mucosa. Loss of pliability. \\
\hline Histological Assessment: & 0 & Absent or rare neutrophil \\
Neutrophil infiltrate & 1 & Moderate or severe neutrophil infiltrate \\
\hline Histological Assessment: & 0 & Ulceration absent \\
Ulceration & 1 & Ulceration present \\
\hline
\end{tabular}

thiobarbituric acid (TBA) reaction proposed by Kohn and Liversedge [25] was used. Tissue samples were defrosted, weighed, and a volume equivalent to five times the weight of TRIS $0.01 \mathrm{M} / \mathrm{pH} 7.4$ buffer solution was then added. Tissue samples were homogenized in an ice bath four times, for 30 seconds each, and subsequently centrifuged for 5 minutes at $10,000 \mathrm{rpm}$, at $4^{\circ} \mathrm{C}$. The protein content of the homogenate was determined by the coomassie brilliant blue (CBB) procedure, as described by Kohn and Liversedge [25]. Briefly, the CBB reactant interacts with protein, enabling its quantification by using a standard albumin curve with known concentrations.

For MDA measurement, 400 micro liters of the centrifuged homogenate supernatant were collected and added to $1 \mathrm{ml}$ of $20 \%$ trichloroacetic acid and $400 \mathrm{ml}$ of $1.6 \%$ thiobarbituric acid.

The mixture was incubated for 30 minutes at $95^{\circ} \mathrm{C}$. Lipids were extracted by adding n-butanol $(1.6 \mathrm{ml})$ and stirring vigorously. The sample was again centrifuged for 10 minutes at $3000 \mathrm{rpm}$.

Absorbance of the organic layer was determined through reading at 510,532, and $560 \mathrm{~nm}$. The following equation, proposed to minimize the interference of both heme pigments and hemoglobin in the measurement of MDA [20,24], was used:

$$
\begin{aligned}
& \text { MDA532 } \\
= & 1.22[(\mathrm{~A} 532)-(0.56)(\mathrm{A} 510)+(0.44)(\mathrm{A} 560)] .
\end{aligned}
$$

The calibration curve was drawn with $1,3,3$ tetramethoxypropane (also known as malondialdehyde bis. MDA levels were calculated and expressed in nmol MDA/mg of protein.

\subsection{Statistical Analysis}

The Kappa coefficient (k) was calculated to determine inter-examiner agreement for clinical assessments of OM. Qualitative variables (clinical and histological scoring of mucositis severity) were compared using the Pearson's
Chi square test and the Fisher test. Quantitative variables (MDA levels) were compared using the analysis of variance (ANOVA). All statistical analyses were performed with a significance level of $5 \%(\alpha=0.05)$.

\section{RESULTS}

\subsection{Clinical Evaluation of OM}

There was excellent inter-examiner agreement on the clinical assessment of OM (Kappa $=0.86$ for Group 1 (glycine supplementation) and 0.94 for Group 2 (controls). These data demonstrate that there was adequate calibration for evaluation of the clinical characteristics of $\mathrm{OM}$. The mucositis induction protocol consistently caused erythema, hemorrhage and ulceration in the right cheek pouch of all animals. Thus, all animals in both groups were scored as having Grade 3 mucositis on day 3 (Figure 1). However, by day 7, there was a marked reduction in Clinical mucositis severity in most animals in the glycine group, with the majority showing healing of ulcerations. In comparison, the clinical mucositis severity in control animals stayed the same or worsened (Table 2). This difference between groups was clinically and statistically significant $(\mathrm{p}<0.001)$.

\subsection{Histological Evaluation of OM}

Histopathological findings in control animals on day 7 were consistent with those previously described for this animal model and mucositis induction protocol [22]. In general, control animals demonstrated an intense cellular infiltration with prevalence of neutrophils, hemorrhagic areas, severe vascular hyperemia, edema, and ulceration. Focal points of surface bacterial colonization and abscesses were seen (Figure 2). In contrast, the glycine group generally exhibited a less intensive histopathologycal reaction, with discreet vascular hyperemia and slight inflammatory infiltration.

On day $7,100 \%$ of animals in the control group had a 


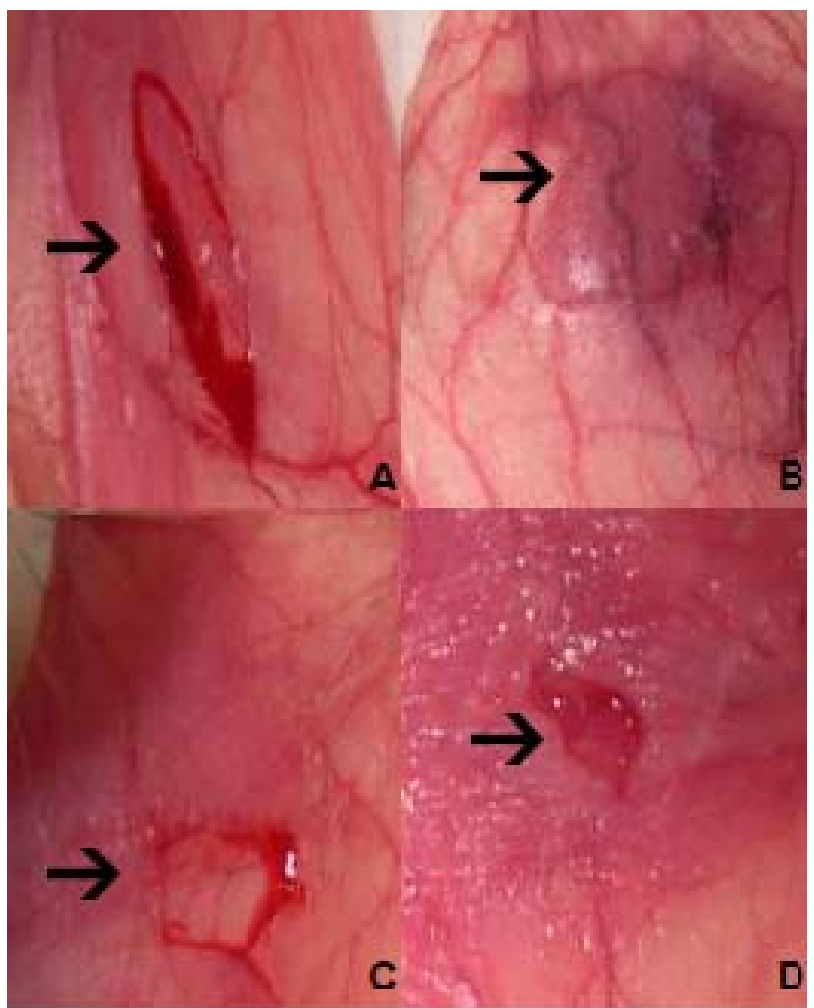

Figure 1. A representative Photographs of the cheek pouch of hamsters at magnification $\times 400$. Glycine group: (A) Day 3 (ulcer present) and (B) Day 7 (re-epithelization of the mucosa). Control group representatives: (C) Day 3 (ulcer present) and (D) Day 7 (persistent ulcer).

Table 2. Clinical evaluation of oral mucositis.

\begin{tabular}{cccccccc}
\hline Day & Group & \multicolumn{5}{c}{ Mucositis grade } \\
\hline \multirow{3}{*}{3} & Glycine & & & & 20 & & \\
& Control & & & & 20 & \\
7 & Glycine $^{*}$ & 1 & 13 & 3 & 3 & & \\
& Control $^{*}$ & & & & 16 & 4 \\
\hline
\end{tabular}

The table represents quantification of data represents number of animals with each grade of oral mucositis at each time-point. ${ }^{*} \mathrm{p}<0.001$.

moderate-severe neutrophil infiltrate (grade 1), as compared to only $25 \%$ of animals in the glycine group (Table 3). The remaining $75 \%$ of animals in the glycine group demonstrated minimal neutrophil infiltrate (grade 0$)(p<$ 0.001 ). On day $7,100 \%$ of animals in the control group demonstrated microscopic ulceration (grade 1), as compared to only $35 \%$ of the animals in the glycine group (Table 3). The remaining $65 \%$ of animals in the glycine group demonstrated re-epithelization and healing, with absence of microscopic ulceration (grade 0$)(p<0.001)$.

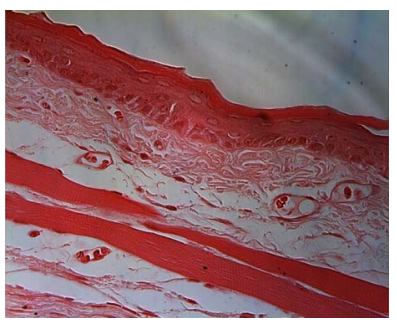

(a)

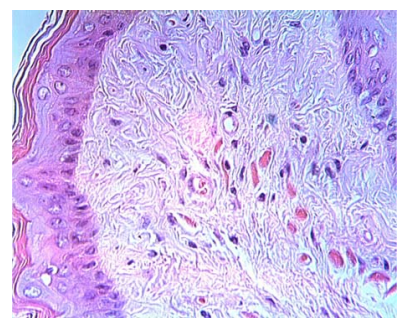

(b)

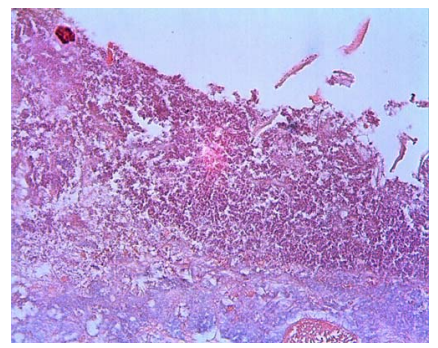

(c)

Figure 2. A representative photomicrograph of hamster oral mucosa on day 7 at magnification $\times 400$, demonstrating ulceration and inflammatory infiltration in epithelial cells. Glycine group representative: (a) Absence of ulceration and inflammatory infiltration. Control group representatives: (b) Moderate inflammatory infiltration; (c) Intense inflammatory infiltration, ulceration and bacterial colonization.

Table 3. Histological evaluation of oral mucositis (Day 7).

\begin{tabular}{ccccc}
\hline \multirow{2}{*}{ Group } & \multicolumn{2}{c}{ Neutrophil Infiltrate } & \multicolumn{2}{c}{ Ulceration } \\
\cline { 2 - 5 } & Grade 0 $^{*}$ & ${\text { Grade } \mathbf{1}^{*}}^{*}$ & Grade 0 $^{*}$ & Grade 1 $^{\#}$ \\
\hline Glycine & 15 & 5 & 13 & 7 \\
Control & 0 & 20 & 0 & 20 \\
\hline
\end{tabular}

The table represents quantification of data represents number of animals with each grade of neutrophil infiltrate and ulceration, at day 7. ${ }^{*} \mathrm{p}<0.001$ ${ }_{\mathrm{p}} \mathrm{p}<0.001$

\subsection{Measurement of Oxidative Stress: Determination of Malondialdehyde (MDA) Levels}

At day 7, the mean MDA levels in the cheek pouch of animals in the control group were more than 5-fold the MDA levels in the glycine group (Table 4). Treatment with glycine thus significantly reduced this marker of lipid peroxidation and free radical production $(\mathrm{p}<0.001)$.

\section{DISCUSSION}

$\mathrm{OM}$ is a complex process involving not only direct cell injury caused by chemotherapy or radiation, but also a complex cascade of biological events [10]. The process begins with clonogenic cell death and the release of reactive oxygen species (ROS), progressing through a series of steps in which multiple biological pathways are activated and amplified, culminating in ulcer development, and finally healing [4]. Investigations into the patho- 
Table 4. Measurement of oxidative stress: Determination of malondialdehyde (MDA) levels (Day 7).

\begin{tabular}{cccc}
\hline \multirow{2}{*}{ Group } & \multicolumn{3}{c}{ Nmol MDA/mg protein } \\
\cline { 2 - 4 } & Mean & Standard Deviation & $\mathbf{N}$ \\
\hline Glycine & $0.185^{*}$ & 0.118 & 20 \\
Control & $1.085^{*}$ & 0.225 & 20 \\
\hline
\end{tabular}

${ }^{*} \mathrm{p}<0.001$.

genesis of OM show the importance of the inflammatory response, which includes the involvement of many different inflammatory mediators including nuclear factor kappa B (NF- $\kappa \mathrm{B})[12,26,27]$, cytokines such as tumor necrosis factor-alpha (TNF- $\alpha$ ), interleukin-1 beta (IL-1 $\beta$ ), interleukin-6 (IL-6) and platelet activating factor (PAF) $[1,22,28,29]$, as well as the cyclooxygenase pathway [1, $23,26,30]$.

Therefore, there has been significant interest in the evaluation of anti-inflammatory strategies for the amelioration of oral mucositis [31]. Glycine is a simple nonessential amino acid that acts as an inhibitory neurotransmitter in the central nervous system (CNS), via a glycine-gated chloride channel (GlyR) [32]. Outside of the CNS, glycine had been presumed to be independently biologically neutral for a long time, functioning only as a building block for proteins. More recently, however, evidence has accumulated indicating that glycine possesses anti-inflammatory properties [19]. Our current results support this anti-inflammatory role for glycine. In a wellaccepted animal model of chemotherapy-induced OM, we found that glycine supplementation significantly reduced the severity of clinical mucositis. The attenuated clinical severity was accompanied by a marked reduction in neutrophil infiltrate, which suggests that glycine suppressed the inflammatory response associated with mucositis. Furthermore, glycine also reduced the production of damaging free radicals, as measured by MDA levels. MDA is a final product of lipid peroxidation and a wellestablished measure of the level of free radicals in intestinal tissue $[20,24]$.

The anti-inflammatory effects of glycine are believed to be mediated, at least in part, due to its mechanism of action in the cell membrane where it activates the chloride channel that stabilizes or hyperpolarizes the membrane potential [33]. Glycine blocks the increase of intracellular calcium which stimulates the formation of the cytokine cascade, inhibiting cells that activate the inflamematory process, probably by blocking activation of NF$\kappa \mathrm{B}$ and TNF- $\alpha$ [34], decreasing the formation of free radicals and other toxic mediators [35]. The free radical nitric oxide and/or its derivatives can cause lipid peroxidation [36], oxidation of protein sulfhydryls [37] and nitration of tyrosine residues on a variety of proteins, including inactivation of enzymes and/or receptors [38-40]. These effects result in tissue injury, which can lead to an excessive local amplification of the inflammatory response.

Mikalauskas et al. demonstrated that glycine decreased chemotherapy-induced liver injury, accompanied by a significant reduction in inducible nitric oxide synthase [19]. Our finding of reduced MDA levels and neutrophil infiltrates in glycine-treated animals is consistent with a role for glycine in reducing lipid peroxidation, free radical formation, and the subsequent tissue injuryand inflammatory response. In a rat model of rheumatoid arthritis, glycine supplementation reduced joint swelling, accompanied by a reduction in TNF- $\alpha$, inflammatory cell infiltrate and edema [41]. Furthermore, in a rat model of ischemia-reperfusion injury, glycine administration resulted in increased mucosal viability and thickness, likely mediated via a down-regulation of cellular apoptosis [20, 42]. Another possible mechanism is derived from the fact that glycine participates in the formation of a third of the structure of collagen. Thus, glycine supplementation may result in increased basement membrane stability [43]. Collectively, these studies point to several mechanisms whereby glycine may be beneficial in ameliorating chemotherapy-induced mucosal injury.

\section{CONCLUSION}

In conclusion, glycine supplementation significantly reduced chemotherapy-induced oral, mucosal injury, neutrophil infiltrate and free radical production in an animal model.

\section{REFERENCES}

[1] Sonis, S.T., Elting, L.S., Keefe, D., Peterson, D.E. and Schubert, M.M. (2004) Perspectives on cancer therapyinduced mucosal injury. Cancer, 100, 1005-2025. doi:10.1002/cncr.20162

[2] Rubenstein, E.B., Peterson, D.E., Schubert, M.M., et al. (2004) Clinical practice guidelines for the prevention and treatment of cancer therapy-induced oral gastrointestinal mucositis. Cancer, 100, 2026-2046. doi: $10.1002 /$ cncr. 20163

[3] Lalla, R.V. and Peterson, D.E. (2005) Oral mucositis. Dental Clinics of North America, 49, 167-184. doi:10.1016/j.cden.2004.07.009

[4] Sonis, S.T. (2004) The pathobiology of mucositis. Nature Reviews Cancer, 4, 277-284. doi:10.1038/nrc1318

[5] Sonis, S.T., Oster, G., Fuchs, H., et al. (2001) Oral mucositis and the clinical and economic outcomes of hematopoetic-stem cells transplantation. Journal of Clinical Oncology, 19, 2201-2205.

[6] Elting, L.S., Keefe, D.M., Sonis, S.T., et al. (2008) Burden of illness head and neck writing committee. Patientreported measurements of oral mucositis in head and neck cancer patients treated with radiotherapy with or without chemotherapy: Demonstration of increased frequency, severity, resistance to palliation, and impact on quality of 
life. Cancer, 113, 2704-2713. doi:10.1002/cncr.23898

[7] Lalla, R.V. (2011) Translating mucositis research from animal models to humans: Challenges and opportunities. The Journal of Supportive Oncology, 9, 169-175. doi:10.1016/j.suponc.2011.04.011

[8] Zanin, T., Zanin, F., Carvalhosa, A.A., Castro, P.H.S., Pacheco, M.T., Zanin, I.C.J., et al. (2010) Use of 660-nm diode laser in prevention and treatment of human oral mucositis induced by radiotherapy and chemotherapy. Photomedicine and Laser Surgery, 28, 233-237. doi:10.1089/pho.2008.2242

[9] Bensadoun, R.J. (2006) Low level laser therapy (LLLT): A new paradigm in themanagement of cancer therapyinduced mucositis. Indian Journal of Medical Research, 124, 375-378.

[10] Sonis, S.T. (2010) New thoughts on the initiation of mucositis. Oral Diseases, 16, 597-600. doi:10.1111/j.1601-0825.2010.01681.x

[11] Haque, J.A., McMahan, R.S., Campbell, J.S., et al. (2011) Attenuated progression of dietinduced steatohepatitis in glutathione-deficient mice. Laboratory Investigation, 90, 1704-1717. doi:10.1038/labinvest.2010.112

[12] Sonis, S.T. (2002) The biologic role for nuclear factorkappaB in disease and its potential involvement in mucosal injury associated with anti-neoplastic therapy. Critical Reviews in Oral Biology \& Medicine, 13, 380-389. doi:10.1177/154411130201300502

[13] Zhong, Z., Wheeler, M.D., Li, X., et al. (2003) L-glycine: A novel anti-inflammatory, immunomodulatory, and cytoprotective agent. Current Opinion in Clinical Nutrition \& Metabolic Care, 6, 229-240. doi:10.1097/00075197-200303000-00013

[14] Wheeler, M., Stachlewitz, R.F., Yamashina, S., Ikejima, K., Morrow, A.L. and Thurman, R.G. (2000) Glycinegated chloride channels in neutrophils attenuate calcium influx and superoxide production. FASEB Journal, 14, 476-484.

[15] Qu, W., Ikejima, K, Zhong, Z., Waalkes, M.P. and Thurman, R.G. (2002) Glycine blocks the increase in intracellular free $\mathrm{Ca} 2$ due to vasoactive mediators in hepatic parenchymal cells. American Journal of Physiology Gastrointestinal and Liver Physiology, 283, G1249-G1256.

[16] Bilzer, M., Baron, A., Schauer, R., Steib, C., Ebensberger, S. and Gerbes, A.L. (2002) Glutathione treatment protects the rat liver against injury after warm ischemia and Kupffer cell activation. Digestion, 66, 49-57. doi:10.1159/000064415

[17] Stoffels, B., Turler, A., Schmidt, J., et al. (2011) Antiinflammatory role of glycine in reducing rodent postoperative inflammatory ileus. Neurogastroenterology \& Motility, 23, 76-78. doi:10.1111/j.1365-2982.2010.01603.x

[18] Neyrinck, A.M., Margagliotti, S. and Delzenne, N.M. (2005) Insight into the involvement of Kupffer cell-derived mediators in the hepatoprotective effect of glycine upon inflammation: study on rat precision-cut liver slices. Inflammation Research, 54, 106-112.

[19] Mikalauskas, S., Mikalauskiene, L., Bruns, H., et al. (2011) Dietary glycine protects from chemotherapy-induced hepatotoxicity. Amino Acids, 40, 1139-1150. doi:10.1007/s00726-010-0737-6
[20] Jacob, T., Ascher, E., Hingorani, A. and Kallakuri, S. (2003) Glycine prevents the induction of apoptosis attributed to mesenteric ichemial-reperfusion injury in a rat model. Surgery, 134, 457-466. doi:10.1067/S0039-6060(03)00164-8

[21] Sonis, S.T., Tracey, C., Shlar, G., Jenson, J., Florine, D. and Almeida, C. (1990) An animal model for mucositis induced by cancer chemotherapy. Oral Surgery, Oral Medicine, Oral Pathology, 69, 437-443. doi:10.1016/0030-4220(90)90376-4

[22] Sonis, S.T., Peterson, R.L., Edwards, L.J., Lucey, C.A., et al. (2000) Defining mechanisms of action of interleukin-11 on the progression of radiation-induced oral mucosites in hamsters. Oral Oncology, 36, 373-81. doi:10.1016/S1368-8375(00)00012-9

[23] Lopes, N.N., Plapler, H., Chavantes, M.C., et al. (2010) Cyclooxygenase- 2 and vascular endothelial growth factor expression in 5-fluorouracil-induced oral mucositis in hamsters: Evaluation of two low-intensity laser protocols. Support Care Cancer, 7, 1409-1415.

[24] Okur, H., Küçükaydin, M., Köse, K., Kontas, O., Dogan, P. and Kazez, A. (1995) Hypoxia-induced necrotizing enterocolitis in the immature rat: the role of lipid peroxidation and management by vitamin E. Journal of Pediatric Surgery, 30, 1416-1432. doi:10.1016/0022-3468(95)90395-X

[25] Kohn, H.I. and Liversedge, M. (1994) On a new aerobic metabolite whose production by brain is inhibited by apomorphine, emetine, ergotamine, epinephrine and manadione. Journal of Pharmacology and Experimental Therapeutics, 82, 292-300.

[26] Logan, R.M., Gibson, R.J., Sonis, S.T., et al. (2007) Nuclear factorkappaB (NF-happaB) and cycloxygenase-2 $(\mathrm{COX}-2)$ expression in oral mucosa following cancer chemotherapy. Oral Oncology, 43, 395-401. doi:10.1016/j.oraloncology.2006.04.011

[27] Yeoh, A.S., Gibson, R.J., Yeoh, E.E., et al. (2007) A novel animal model to investigate fractionated radiotherapyinduced alimentary mucositis: The role of apoptosis, p53, nuclear factor-kappaB, COX-1, and COX-2. Molecular Cancer Therapeutics, 6, 2319-2327. doi:10.1158/1535-7163.MCT-07-0113

[28] Kuenen, B.C., Levi, M., Meijers, J.C., et al. (2003) Potential role of platelets in endothelial damage during treatment with cisplatin-gemcitabine, and the angiogenesis inhibitorSU5416. Journal of Clinical Oncology, 21, 2192-2198. doi:10.1200/JCO.2003.08.046

[29] Logan, R.M., Stringer, A.M., Bowen, J.M., et al. (2008) Serum levels of NF-kB and proinflammatory cytokines following administration of mucotoxic drugs. Cancer Biology \& Therapy, 7, 1139-1145. doi:10.4161/cbt.7.7.6207

[30] Lalla, R.V., Pilbeam, C.C., Walsh, S.J., et al. (2010) Role of the cyclooxygenase pathway in chemotherapy-induced oral mucositis: A pilot study. Support Care Cancer, 18, 95-103. doi:10.1007/s00520-009-0635-1

[31] Lalla, R.V., Schubert, M.M., Bensadoun, R.J. and Keefe, D, (2006) Anti-inflammatory agents in the management of alimentary mucositis. Support Care Cancer, 14, 558565. doi:10.1007/s00520-006-0050-9 
[32] Froh, M., Thurman, R.G. and Wheeler, M.D. (2002) Molecular evidence for a glycine-gated chloride channel in macrophages and leukocytes. American Journal of Physiology Gastrointestinal and Liver Physiology, 283, G856G863.

[33] Hartog, A.L. (2007) Anti-inflammatory effects of orally ingested lactoferrin and glycine in different zymosan-induced inflammation models: Evidence for synergistic activit. International Immunopharmacology, 7, 1784-1792. doi:10.1016/j.intimp.2007.09.019

[34] Wagner, A.F., Frey, M., Neugebauer, F.A., Schäfer, W. and Knappe, J. (1992) The free radical in pyruvate formate-lyase is located on glycine-734. Proceedings of the National Academy of Sciences, 89, 996-1000. doi:10.1073/pnas.89.3.996

[35] Mauriz, B., Matilla, J., Culebras, M., González, P. and González-Gallego, J. (2001) Dietary glycine inhibits activation of nuclear factor kappa B and prevents liver injury in hemorrhagic shock in the rat. Free Radical Biology \& Medicine, 31, 1236-1244. doi:10.1016/S0891-5849(01)00716-X

[36] Rubbo, H., Radi, L., Trugillo, M., et al. (1994) Nitric oxide regulation of superoxide and peroxynitrite-dependent lipid peroxidation. Formation of novel nitrogen-containing oxidized lipid derivatives. The Journal of Biological Chemistry, 296, 26066-26075.

[37] Radi, R., Beckman, J.S., Bush, K.M., et al. (1991) Peroxynitrite oxidation of sulfhydryls. The cytotoxic poten- tial of superoxide and nitric oxide. The Journal of Biological Chemistry, 266, 4244-4250.

[38] Beckman, J.S. (1996) Oxidative damage and tyrosine nitration from peroxynitrite. Chemical Research in Toxicology, 9, 836-844. doi:10.1021/tx9501445

[39] Beckman, J.S. and Koppenol, W.H. (1996) Nitric oxide, superoxide, and peroxynitrite: The good, the bad, and ugly. American Journal of Physiology, 271, C1424-C1437.

[40] Szabo, C. (1996) The pathophysiological role of peroxynitrite in shock, inflammation, and ischemia-reperfusion injury. Shock, 6, 79-88. doi:10.1097/00024382-199608000-00001

[41] Li, X., Bradford, B.U., Wheeler, M.D., et al. (2001) Dietary glycine prevents peptidoglycan polysaccharideinduced reactive arthritis in the rat: Role for glycine-gated chloride channel. Infection and Immunity, 69, 5883-5891. doi:10.1128/IAI.69.9.5883-5891.2001

[42] Kallakuri, S., Pagala, M., Gade, P., et al. (2003) Protective effect of glycine in mesenteric ischemia and reperfusion injury in a rat model. Journal of Vascular Surgery, 38, 1113-1120. doi:10.1016/S0741-5214(03)00939-X

[43] Pöschl, E., Schlötzer-Schrehardt, U., Brachvogel, B., Saito, K., Ninomiya, Y. and Mayer, U. (2004) Collagen IV is essential for basement membrane stability but dispensable for initiation of its assembly during early development. Epub, 131, 1619-1628. 